\title{
Conformal Hamiltonian Mechanical Equations on Contact 9- Manifolds
}

\author{
Ibrahim Omer Ahmed Albudawe ${ }^{1^{*}}$, Ibrahim Yousif .I. Abad Alrhman 1,2 \\ ${ }^{1}$ Department of Mathematics, College of Science - Jouf University. Saudi Arabia \\ ${ }^{2}$ Department of Mathematics, Faculty of Education. West Kordufan University, Sudan
}

Received: 20/11/2020

Accepted: 04/01/2021

Published: 20/06/2021

\begin{abstract}
In this study, we concluded the Hamiltonian equations on $\left(\mathcal{M}^{9}, \lambda, \mathrm{X}_{\mathrm{H}}\right)$, being a model. Finally introduce, some geometrical and physical results on the related mechanic systems have been discussed. $n$ classical mechanics we can describe the state of a system by specifying its Lagrangian as a function of the coordinates and their time rates of change. Hamilton realized that Lagrange's equations of motion were equivalent to a variational principle.
\end{abstract}

Keywords: Differential geometry, Contact 9- Manifolds, Hamiltonian Dynamics

\section{Introduction ${ }^{1}$}

\subsection{Historical Background}

Hamiltonian Mechanics was introduced in 1833 by the Irish mathematician William Rowan Hamilton. As a man inspired with knowledge at a very young age Hamilton was able to accomplish many tasks in only the 60 years he lived. While there, he studied optics, classical mechanics, dynamic methods, quaternion's, and many other topics. Hamilton remained active in the mathematical society until his death in 1865 from a severe case of gout, (1). Modern Differential Geometry is a suitable frame for studying Hamiltonian formalisms of Classical Mechanics. To show this, it is possible to find many articles and books in the relevant fields. It is well-known that the dynamics of Hamiltonian systems is characterized by a convenient vector field $\mathrm{X}$ defined on the tangent and cotangent bundles which are phase-spaces of velocities and momentum of a given configuration manifold. If $\mathcal{M}$ is an m-dimensional configuration manifold and $H: T \mathcal{M} \rightarrow R$ is a regular Lagrangian function, then there is a unique vector field $\mathrm{X}$ on $T \mathcal{M}$ such that

$i_{X} \omega=$

$d H$

where $\omega$ is the Symplectic form and $\mathrm{H}$ stands for Hamiltonian function. The paths of the so called Hamiltonian vector field $X_{H}$ are the solutions of the Hamiltonian equations. The triple, either $\left(T^{*} \mathcal{M}, \omega, X_{H}\right)$ or $\left(T^{*} \mathcal{M}, \omega, H\right)$, is called Hamiltonian system on the cotangent bundle $T^{*} \mathcal{M}$ fixed with Symplectic form $\omega(2)$

\section{Complex on Contact 9-manifolds \\ 2.1 Definition (3)}

The contact 9-manifold $\left(M^{9}, \alpha\right)$, given a horizontal 2-form $\omega$.

\subsection{Theorem}

A conformal manifold is a differentiable manifold equipped with an equivalence class of Riemann metric tensors, in which twof $_{1}$ metricsf $_{2}$ and are equivalent if and only if

$f_{2}=B^{2} f_{1}$

where is a smooth positive function. $\mathrm{B}>0$

\subsection{Theorem 1}

A conformal transformation is a change of coordinates such that the metric changes by $\sigma^{\mathrm{a}} \rightarrow \sigma^{\mathrm{b}}$

$\mathrm{f}_{\mathrm{ab}}(\sigma) \rightarrow \mathrm{B}^{2}(\sigma) \mathrm{f}_{\mathrm{ab}}(\sigma)$

\subsection{Proposition}

Suppose that $\left\{x_{1}, x_{2}, x_{3}, x_{4}, x_{5}, x_{6}, x_{7}, x_{8}, \theta\right\}$, be a real coordinate system on $(\mathcal{M}, J)$. Then we denote by

$$
\begin{aligned}
& \left\{\frac{\partial}{\partial x_{1}}, \frac{\partial}{\partial x_{2}}, \frac{\partial}{\partial x_{3}}, \frac{\partial}{\partial x_{4}}, \frac{\partial}{\partial x_{5}}, \frac{\partial}{\partial x_{6}}, \frac{\partial}{\partial x_{7}}, \frac{\partial}{\partial x_{8}}, \theta\right\} \\
& \left\{d x_{1}, d x_{2}, d x_{3}, d x_{4}, d x_{5}, d x_{6}, d x_{7}, d x_{8}, \theta\right\}
\end{aligned}
$$

\subsection{Proposition}

The following expressions are given. The dual form $J^{*}$ of the above $J$ is as follows:

$$
\begin{gathered}
\mathrm{J}^{*}\left(\mathrm{dx}_{1}\right)=\mathrm{B}^{2} \cos \theta \mathrm{dx}_{5}+\mathrm{B}^{2} \sin \theta \mathrm{dx}_{6}, \mathrm{~J}^{*}\left(\mathrm{dx}_{2}\right) \\
=-\mathrm{B}^{2} \cos \theta \mathrm{dx}_{6}+\mathrm{B}^{2} \sin \theta \mathrm{dx}_{5} \\
\begin{array}{r}
\mathrm{J}^{*}\left(\mathrm{dx}_{3}\right)=\mathrm{B}^{2} \cos \theta \mathrm{dx}_{7}+\mathrm{B}^{2} \sin \theta \mathrm{dx}_{8}, \mathrm{~J}^{*}\left(\mathrm{dx}_{4}\right) \\
=-\mathrm{B}^{2} \cos \theta \mathrm{dx}_{8}+\sin \theta \mathrm{dx}_{7}
\end{array} \\
\begin{array}{r}
\mathrm{J}^{*}\left(\mathrm{dx}_{5}\right)=-\mathrm{B}^{-2} \cos \theta \mathrm{dx}_{7}-\mathrm{B}^{-2} \sin \theta \mathrm{dx}_{8}, \\
\mathrm{~J}^{*}\left(\mathrm{dx}_{6}\right)=\mathrm{B}^{-2} \cos \theta \mathrm{dx}_{8}-\mathrm{B}^{-2} \sin \theta \mathrm{dx_{7 }}
\end{array}
\end{gathered}
$$

Corresponding author: Ibrahim Omer Ahmed Albudawe, Department of Mathematics, College of Science -Aljouf University, Saudi Arabia Email: iyibrahimi@gmail.com 
$\mathrm{J}^{*}\left(\mathrm{dx}_{7}\right)=-\mathrm{B}^{-2} \cos \theta d \mathrm{dx}_{3}-\mathrm{B}^{-2} \sin \theta \mathrm{dx}_{4}, \mathrm{~J}^{*}\left(\mathrm{dx}_{8}\right)=$ $\mathrm{B}^{-2} \cos \theta \mathrm{dx}_{4}-\mathrm{B}^{-2} \sin \theta \mathrm{dx}_{3}$

\subsection{Definition}

A Hamiltonian system is a triple $(\mathcal{M} ; \xi)$, where $(\omega ; L)$ is a Symplectic manifold and $H \in C^{\infty}(\mathcal{M})$ is a function, called the Hamiltonian function.

\subsection{Theorem}

Let $\mathcal{M}$ be m-real dimensional configuration manifold .A tensor field $J$ on $T^{*} \mathcal{M}$ is called an almost complex structure on $T^{*} \mathcal{M}$ if at every point $\mathrm{p}$ of $T^{*} \mathcal{M}, \mathrm{J}$ is endomorphism of the tangent space $T_{p}{ }^{*}(\mathcal{M})$ such that $J^{2}=-1$ are complex is

$J^{* 2}\left(d x_{i}\right)=-d x_{i} \quad, \quad i=1,2,3,4,5,6,7,8$

$J^{* 2}$ is called almost complex manifold

\section{Hamiltonian Dynamical Systems \\ 3.1 Definition}

A Hamiltonian function for a Hamiltonian vector field $X$ on $\mathcal{M}$ is a smooth function $H: \mathcal{M} \rightarrow R$ such that $\mathrm{i}_{\mathrm{X}_{\mathrm{H}}} \omega=\mathrm{dH}$.

\subsection{Definition}

A Hamiltonian system is a triple $(M ; \omega ; H)$, where $(\omega ; H)$ is a Symplectic manifold and $H \in C^{\infty}(M)$ is a function, called the Hamiltonian function. Suppose that an almost real structure, a Liouville form and a1-form on $\mathrm{T}^{*} \mathcal{M}$ are shown by $\Phi^{*}, \lambda$ and $\omega$, respectively. Then we have:

$$
\begin{gathered}
\omega=\frac{1}{2}\left(x_{1} d x_{1}+x_{2} d x_{2}+x_{3} d x_{3}+x_{4} d x_{4}+x_{5} d x_{5}+x_{6} d x_{6}\right. \\
\left.+x_{7} d x_{7}+x_{8} d x_{8}\right)
\end{gathered}
$$

and

$\lambda$

$$
\begin{aligned}
& =\frac{1}{2}\left(\mathrm{x}_{1} J^{*}\left(d x_{1}\right)+\mathrm{x}_{2} J^{*}\left(d x_{2}\right)+\mathrm{x}_{3} J^{*}\left(d x_{3}\right)+\mathrm{x}_{4} J^{*}\left(d x_{4}\right)\right. \\
& +\mathrm{x}_{5} J^{*}\left(d x_{5}\right)+\mathrm{x}_{6} J^{*}\left(d x_{6}\right)+\mathrm{x}_{7} J^{*}\left(d x_{7}\right) \\
& \left.+\mathrm{x}_{8} J^{*}\left(d x_{8}\right)\right)
\end{aligned}
$$

We substitute equation (2) in equation (3) we get:

$$
\begin{aligned}
\lambda=\Phi^{*}(\omega)=\frac{1}{2}\left[\mathrm{x}_{1}\right. & \left.\mathrm{B}^{2} \cos \theta d x_{5}+\mathrm{B}^{2} \sin \theta d x_{6}\right) \\
& +\mathrm{x}_{2}\left(-\mathrm{B}^{2} \cos \theta d x_{6}+\mathrm{B}^{2} \sin \theta d x_{5}\right) \\
& +\mathrm{x}_{3}\left(\mathrm{~B}^{2} \cos \theta d x_{7}+\mathrm{B}^{2} \sin \theta d x_{8}\right) \\
& +\mathrm{x}_{4}\left(-\mathrm{B}^{2} \cos \theta d x_{8}+\mathrm{B}^{2} \sin \theta d x_{7}\right) \\
& +\mathrm{x}_{5}\left(-\mathrm{B}^{-2} \cos \theta d x_{7}-\mathrm{B}^{-2} \sin \theta d x_{8}\right) \\
& +\mathrm{x}_{6}\left(\mathrm{~B}^{-2} \cos \theta d x_{8}-\mathrm{B}^{-2} \sin \theta d x_{7}\right) \\
& +\mathrm{x}_{7}\left(-\mathrm{B}^{-2} \cos \theta d x_{3}-\mathrm{B}^{-2} \sin \theta d x_{4}\right) \\
& \left.+\mathrm{x}_{8}\left(\mathrm{~B}^{-2} \cos \theta d x_{4}-\mathrm{B}^{-2} \sin \theta d x_{3}\right)\right]
\end{aligned}
$$

differential of $\lambda$

$$
\begin{aligned}
& \phi=-\mathrm{d} \lambda= \\
& -\mathrm{d}\left(\frac { 1 } { 2 } \left[\mathrm{x}_{1}\left(\mathrm{~B}^{2} \cos \theta d x_{5}+\mathrm{B}^{2} \sin \theta d x_{6}\right)\right.\right. \\
& +\mathrm{x}_{2}\left(-\mathrm{B}^{2} \cos \theta d x_{6}+\mathrm{B}^{2} \sin \theta d x_{5}\right) \\
& +\mathrm{x}_{3}\left(\mathrm{~B}^{2} \cos \theta d x_{7}+\mathrm{B}^{2} \sin \theta d x_{8}\right) \\
& +\mathrm{x}_{4}\left(-\mathrm{B}^{2} \cos \theta d x_{8}+\mathrm{B}^{2} \sin \theta d x_{7}\right) \\
& +\mathrm{x}_{5}\left(-\mathrm{B}^{-2} \cos \theta d x_{7}-\mathrm{B}^{-2} \sin \theta d x_{8}\right) \\
& +\mathrm{x}_{6}\left(\mathrm{~B}^{-2} \cos \theta d x_{8}-\mathrm{B}^{-2} \sin \theta d x_{7}\right) \\
& +\mathrm{x}_{7}\left(-\mathrm{B}^{-2} \cos \theta d x_{3}-\mathrm{B}^{-2} \sin \theta d x_{4}\right) \\
& \left.\left.+\mathrm{x}_{8}\left(\mathrm{~B}^{-2} \cos \theta d x_{4}-\mathrm{B}^{-2} \sin \theta d x_{3}\right)\right]\right)
\end{aligned}
$$

$$
\begin{aligned}
& =\frac{1}{2}\left(\left[\frac{\mathrm{dx}_{1}}{\mathrm{dx}_{1}}\left(\mathrm{~B}^{2} \cos \theta d x_{1} \wedge d x_{5}+\mathrm{B}^{2} \sin \theta d x_{1} \wedge d x_{6}\right)\right.\right. \\
& +\frac{\mathrm{dx}_{2}}{\mathrm{dx}_{2}}\left(-\mathrm{B}^{2} \cos \theta d x_{2} \wedge d x_{6}\right. \\
& \left.+\mathrm{B}^{2} \sin \theta d x_{2} \wedge d x_{5}\right) \\
& +\frac{\mathrm{dx}_{3}}{\mathrm{dx}_{3}}\left(\mathrm{~B}^{2} \cos \theta d x_{3} \wedge d x_{7}\right. \\
& \left.+\mathrm{B}^{2} \sin \theta d x_{3} \wedge d x_{8}\right) \\
& +\frac{\mathrm{dx}_{4}}{\mathrm{dx}_{4}}\left(-\mathrm{B}^{2} \cos \theta d x_{4} \wedge d x_{8}\right. \\
& \left.+\mathrm{B}^{2} \sin \theta d x_{4} \wedge d x_{7}\right) \\
& +\frac{\mathrm{dx}_{5}}{\mathrm{dx}_{5}}\left(-\mathrm{B}^{-2} \cos \theta d x_{5} \wedge d x_{7}\right. \\
& \left.-\mathrm{B}^{-2} \sin \theta d x_{5} \wedge d x_{8}\right) \\
& +\frac{\mathrm{dx}_{6}}{\mathrm{dx}_{6}}\left(\mathrm{~B}^{-2} \cos \theta d x_{6} \wedge d x_{8}\right. \\
& \left.-\mathrm{B}^{-2} \sin \theta d x_{6} \wedge d x_{7}\right) \\
& +\frac{\mathrm{dx}_{7}}{\mathrm{dx}_{7}}\left(-\mathrm{B}^{-2} \cos \theta d x_{7} \wedge d x_{3}\right. \\
& \left.-\mathrm{B}^{-2} \sin \theta d x_{7} \wedge d x_{4}\right) \\
& +\frac{\mathrm{dx}_{8}}{\mathrm{dx}_{8}}\left(\mathrm{~B}^{-2} \cos \theta d x_{8} \wedge d x_{4}\right. \\
& \left.\left.\left.-\mathrm{B}^{-2} \sin \theta d x_{8} \wedge d x_{3}\right)\right]\right) \\
& \phi \\
& =\frac{1}{2}\left(\left[\left(\mathrm{~B}^{2} \cos \theta d x_{1} \wedge d x_{5}+\mathrm{B}^{2} \sin \theta d x_{1} \wedge d x_{6}\right)\right.\right. \\
& +\left(-\mathrm{B}^{2} \cos \theta d x_{2} \wedge d x_{6}+\mathrm{B}^{2} \sin \theta d x_{2} \wedge d x_{5}\right) \\
& +\left(\mathrm{B}^{2} \cos \theta d x_{3} \wedge d x_{7}+\mathrm{B}^{2} \sin \theta d x_{3} \wedge d x_{8}\right) \\
& +\left(-\mathrm{B}^{2} \cos \theta d x_{4} \wedge d x_{8}+\mathrm{B}^{2} \sin \theta d x_{4} \wedge d x_{7}\right) \\
& +\left(-\mathrm{B}^{-2} \cos \theta d x_{5} \wedge d x_{7}-\mathrm{B}^{-2} \sin \theta d x_{5} \wedge d x_{8}\right) \\
& +\left(\mathrm{B}^{-2} \cos \theta d x_{6} \wedge d x_{8}-\mathrm{B}^{-2} \sin \theta d x_{6} \wedge d x_{7}\right) \\
& +\left(-\mathrm{B}^{-2} \cos \theta d x_{7} \wedge d x_{3}-\mathrm{B}^{-2} \sin \theta d x_{7} \wedge d x_{4}\right) \\
& +\left(\mathrm{B}^{-2} \cos \theta d x_{8} \wedge d x_{4}\right. \\
& \left.\left.-\mathrm{B}^{-2} \sin \theta d x_{8} \wedge d x_{3}\right)\right] \text { ) }
\end{aligned}
$$

It is known that if $\phi$ is a closed 2- form on $\mathrm{T}^{*} \mathcal{M}$, then $\phi_{\mathrm{H}}$ is also a symplectic structure on $\mathrm{T}^{*} \mathcal{M}$. If Hamiltonian vector field $\mathrm{X}_{\mathrm{H}}$ associated with Hamiltonian energy $\mathrm{H}$ is given by

$$
\begin{gathered}
\mathrm{X}_{\mathrm{H}}=\mathrm{X}^{1} \frac{\partial}{\partial \mathrm{x}_{1}}+\mathrm{X}^{2} \frac{\partial}{\partial \mathrm{x}_{2}}+\mathrm{X}^{3} \frac{\partial}{\partial \mathrm{x}_{3}}+\mathrm{X}^{4} \frac{\partial}{\partial \mathrm{x}_{4}}+\mathrm{X}^{5} \frac{\partial}{\partial \mathrm{x}_{5}}+\mathrm{X}^{6} \frac{\partial}{\partial \mathrm{x}_{6}} \\
+\mathrm{X}^{7} \frac{\partial}{\partial \mathrm{x}_{7}}+\mathrm{X}^{8} \frac{\partial}{\partial \mathrm{x}_{8}}
\end{gathered}
$$

Calculates a value $\mathrm{X}_{\mathrm{H}}$ and $\phi$

$$
\begin{aligned}
& \phi\left(\mathrm{X}_{\mathrm{H}}\right)= \\
& \quad \frac{1}{2}\left(\left[\left(\mathrm{~B}^{2} \cos \theta d x_{1} \wedge d x_{5}+\mathrm{B}^{2} \sin \theta d x_{1} \wedge d x_{6}\right)\right.\right. \\
& +\left(-\mathrm{B}^{2} \cos \theta d x_{2} \wedge d x_{6}+\mathrm{B}^{2} \sin \theta d x_{2} \wedge d x_{5}\right) \\
& +\left(\mathrm{B}^{2} \cos \theta d x_{3} \wedge d x_{7}+\mathrm{B}^{2} \sin \theta d x_{3} \wedge d x_{8}\right) \\
& +\left(-\mathrm{B}^{2} \cos \theta d x_{4} \wedge d x_{8}+\mathrm{B}^{2} \sin \theta d x_{4} \wedge d x_{7}\right) \\
& +\left(-\mathrm{B}^{-2} \cos \theta d x_{5} \wedge d x_{7}-\mathrm{B}^{-2} \sin \theta d x_{5} \wedge d x_{8}\right) \\
& +\left(\mathrm{B}^{-2} \cos \theta d x_{6} \wedge d x_{8}-\mathrm{B}^{-2} \sin \theta d x_{6} \wedge d x_{7}\right) \\
& +\left(-\mathrm{B}^{-2} \cos \theta d x_{7} \wedge d x_{3}-\mathrm{B}^{-2} \sin \theta d x_{7} \wedge d x_{4}\right) \\
& \left.\left.+\left(\mathrm{B}^{-2} \cos \theta d x_{8} \wedge d x_{4}-\mathrm{B}^{-2} \sin \theta d x_{8} \wedge d x_{3}\right)\right]\right)\left(X^{1} \frac{\partial}{\partial \mathrm{x}_{1}}\right. \\
& +X^{2} \frac{\partial}{\partial \mathrm{x}_{2}}+X^{3} \frac{\partial}{\partial \mathrm{x}_{3}}+X^{4} \frac{\partial}{\partial \mathrm{x}_{4}}+X^{5} \frac{\partial}{\partial \mathrm{x}_{5}}+X^{6} \frac{\partial}{\partial \mathrm{x}_{6}}+X^{7} \frac{\partial}{\partial \mathrm{x}_{7}} \\
& \left.+X^{8} \frac{\partial}{\partial \mathrm{x}_{8}}\right) \\
& \quad \text { And } \\
& \mathrm{i}_{\mathrm{X}_{\mathrm{H}}} \phi=\phi\left(\mathrm{X}_{\mathrm{H}}\right)
\end{aligned}
$$


$\left(\mathrm{B}^{2} \cos \theta d x_{1} \wedge d x_{5}+\mathrm{B}^{2} \sin \theta d x_{1} \wedge d x_{6}\right)\left(X^{1} \frac{\partial}{\partial \mathrm{x}_{1}}+X^{2} \frac{\partial}{\partial \mathrm{x}_{2}}\right.$ $+X^{3} \frac{\partial}{\partial \mathrm{x}_{3}}+X^{4} \frac{\partial}{\partial \mathrm{x}_{4}}+X^{5} \frac{\partial}{\partial \mathrm{x}_{5}}+X^{6} \frac{\partial}{\partial \mathrm{x}_{6}}$ $\left.+X^{7} \frac{\partial}{\partial \mathrm{x}_{7}}+X^{8} \frac{\partial}{\partial \mathrm{x}_{8}}\right)$

$+\left(-\mathrm{B}^{2} \cos \theta d x_{2} \wedge d x_{6}\right.$

$\left.+\mathrm{B}^{2} \sin \theta d x_{2} \wedge d x_{5}\right)\left(X^{1} \frac{\partial}{\partial \mathrm{x}_{1}}+X^{2} \frac{\partial}{\partial \mathrm{x}_{2}}\right.$

$+X^{3} \frac{\partial}{\partial \mathrm{x}_{3}}+X^{4} \frac{\partial}{\partial \mathrm{x}_{4}}+X^{5} \frac{\partial}{\partial \mathrm{x}_{5}}+X^{6} \frac{\partial}{\partial \mathrm{x}_{6}}$

$+X^{7} \frac{\partial}{\partial \mathrm{x}_{7}}$

$\left.+X^{8} \frac{\partial}{\partial \mathrm{x}_{8}}\right)\left(\mathrm{B}^{2} \cos \theta d x_{3} \wedge d x_{7}\right.$

$\left.+\mathrm{B}^{2} \sin \theta d x_{3} \wedge d x_{8}\right)\left(X^{1} \frac{\partial}{\partial \mathrm{x}_{1}}+X^{2} \frac{\partial}{\partial \mathrm{x}_{2}}\right.$

$+X^{3} \frac{\partial}{\partial \mathrm{x}_{3}}+X^{4} \frac{\partial}{\partial \mathrm{x}_{4}}+X^{5} \frac{\partial}{\partial \mathrm{x}_{5}}+X^{6} \frac{\partial}{\partial \mathrm{x}_{6}}$

$\left.+X^{7} \frac{\partial}{\partial \mathrm{x}_{7}}+X^{8} \frac{\partial}{\partial \mathrm{x}_{8}}\right)$

$+\left(-\mathrm{B}^{2} \cos \theta d x_{4} \wedge d x_{8}\right.$

$\left.+\mathrm{B}^{2} \sin \theta d x_{4} \wedge d x_{7}\right)\left(X^{1} \frac{\partial}{\partial \mathrm{x}_{1}}+X^{2} \frac{\partial}{\partial \mathrm{x}_{2}}\right.$

$+X^{3} \frac{\partial}{\partial \mathrm{x}_{3}}+X^{4} \frac{\partial}{\partial \mathrm{x}_{4}}+X^{5} \frac{\partial}{\partial \mathrm{x}_{5}}+X^{6} \frac{\partial}{\partial \mathrm{x}_{6}}$

$\left.+X^{7} \frac{\partial}{\partial \mathrm{x}_{7}}+X^{8} \frac{\partial}{\partial \mathrm{x}_{8}}\right)$

$+\left(-\mathrm{B}^{-2} \cos \theta d x_{5} \wedge d x_{1}\right.$

$\left.-\mathrm{B}^{-2} \sin \theta d x_{5} \wedge d x_{2}\right)\left(X^{1} \frac{\partial}{\partial \mathrm{x}_{1}}+X^{2} \frac{\partial}{\partial \mathrm{x}_{2}}\right.$

$+X^{3} \frac{\partial}{\partial \mathrm{x}_{3}}+X^{4} \frac{\partial}{\partial \mathrm{x}_{4}}+X^{5} \frac{\partial}{\partial \mathrm{x}_{5}}+X^{6} \frac{\partial}{\partial \mathrm{x}_{6}}$

$\left.+X^{7} \frac{\partial}{\partial \mathrm{x}_{7}}+X^{8} \frac{\partial}{\partial \mathrm{x}_{8}}\right)$

$+\left(\mathrm{B}^{-2} \cos \theta d x_{6} \wedge d x_{2}\right.$

$\left.-\mathrm{B}^{-2} \sin \theta d x_{6} \wedge d x_{1}\right)\left(X^{1} \frac{\partial}{\partial \mathrm{x}_{1}}+X^{2} \frac{\partial}{\partial \mathrm{x}_{2}}\right.$

$+X^{3} \frac{\partial}{\partial \mathrm{x}_{3}}+X^{4} \frac{\partial}{\partial \mathrm{x}_{4}}+X^{5} \frac{\partial}{\partial \mathrm{x}_{5}}+X^{6} \frac{\partial}{\partial \mathrm{x}_{6}}$

$\left.+X^{7} \frac{\partial}{\partial \mathrm{x}_{7}}+X^{8} \frac{\partial}{\partial \mathrm{x}_{8}}\right)$

$+\left(-\mathrm{B}^{-2} \cos \theta d x_{7} \wedge d x_{3}\right.$

$\left.-\mathrm{B}^{-2} \sin \theta d x_{7} \wedge d x_{4}\right)\left(X^{1} \frac{\partial}{\partial \mathrm{x}_{1}}+X^{2} \frac{\partial}{\partial \mathrm{x}_{2}}\right.$

$+X^{3} \frac{\partial}{\partial \mathrm{x}_{3}}+X^{4} \frac{\partial}{\partial \mathrm{x}_{4}}+X^{5} \frac{\partial}{\partial \mathrm{x}_{5}}+X^{6} \frac{\partial}{\partial \mathrm{x}_{6}}$

$\left.+X^{7} \frac{\partial}{\partial \mathrm{x}_{7}}+X^{8} \frac{\partial}{\partial \mathrm{x}_{8}}\right)$

$+\left(\mathrm{B}^{-2} \cos \theta d x_{8} \wedge d x_{4}\right.$

$\left.-\mathrm{B}^{-2} \sin \theta d x_{8} \wedge d x_{3}\right)\left(X^{1} \frac{\partial}{\partial \mathrm{x}_{1}}+X^{2} \frac{\partial}{\partial \mathrm{x}_{2}}\right.$

$+X^{3} \frac{\partial}{\partial \mathrm{x}_{3}}+X^{4} \frac{\partial}{\partial \mathrm{x}_{4}}+X^{5} \frac{\partial}{\partial \mathrm{x}_{5}}+X^{6} \frac{\partial}{\partial \mathrm{x}_{6}}$

$\left.+X^{7} \frac{\partial}{\partial \mathrm{x}_{7}}+X^{8} \frac{\partial}{\partial \mathrm{x}_{8}}\right)$

or $\left(d x_{1} \mathrm{~B}^{2} \cos \theta d x_{5}+d x_{1} \mathrm{~B}^{2} \sin \theta d x_{6}-d x_{5} \mathrm{~B}^{2} \cos \theta d x_{1}\right.$

$$
\begin{aligned}
& \left.-d x_{6} \mathrm{~B}^{2} \sin \theta d x_{1}\right)\left(X^{1} \frac{\partial}{\partial \mathrm{x}_{1}}+X^{2} \frac{\partial}{\partial \mathrm{x}_{2}}\right. \\
& +X^{3} \frac{\partial}{\partial \mathrm{x}_{3}}+X^{4} \frac{\partial}{\partial \mathrm{x}_{4}}+X^{5} \frac{\partial}{\partial \mathrm{x}_{5}}+X^{6} \frac{\partial}{\partial \mathrm{x}_{6}} \\
& \left.+X^{7} \frac{\partial}{\partial \mathrm{x}_{7}}+X^{8} \frac{\partial}{\partial \mathrm{x}_{8}}\right)
\end{aligned}
$$

$+\left(d x_{3} \mathrm{~B}^{2} \cos \theta d x_{7}+d x_{3} \mathrm{~B}^{2} \sin \theta d x_{8}\right.$

$-d x_{7} \mathrm{~B}^{2} \cos \theta d x_{8}$

$\left.-d x_{8} \mathrm{~B}^{2} \sin \theta d x_{3}\right)\left(X^{1} \frac{\partial}{\partial \mathrm{x}_{1}}+X^{2} \frac{\partial}{\partial \mathrm{x}_{2}}\right.$

$+X^{3} \frac{\partial}{\partial \mathrm{x}_{3}}+X^{4} \frac{\partial}{\partial \mathrm{x}_{4}}+X^{5} \frac{\partial}{\partial \mathrm{x}_{5}}+X^{6} \frac{\partial}{\partial \mathrm{x}_{6}}$

$\left.+X^{7} \frac{\partial}{\partial \mathrm{x}_{7}}+X^{8} \frac{\partial}{\partial \mathrm{x}_{8}}\right)$

$+\left(-d x_{4} \mathrm{~B}^{2} \cos \theta d x_{8}\right.$

$+d x_{4} \mathrm{~B}^{2} \sin \theta d x_{7}$

$+d x_{8} \mathrm{~B}^{2} \cos \theta d x_{4}$

$\left.+d x_{7} \mathrm{~B}^{2} \sin \theta d x_{4}\right)\left(X^{1} \frac{\partial}{\partial \mathrm{x}_{1}}+X^{2} \frac{\partial}{\partial \mathrm{x}_{2}}\right.$

$+X^{3} \frac{\partial}{\partial \mathrm{x}_{3}}+X^{4} \frac{\partial}{\partial \mathrm{x}_{4}}+X^{5} \frac{\partial}{\partial \mathrm{x}_{5}}+X^{6} \frac{\partial}{\partial \mathrm{x}_{6}}$

$\left.+X^{7} \frac{\partial}{\partial \mathrm{x}_{7}}+X^{8} \frac{\partial}{\partial \mathrm{x}_{8}}\right)$

$+\left(-d x_{5} \mathrm{~B}^{2} \cos \theta d x_{1}\right.$

$-d x_{5} \mathrm{~B}^{2} \sin \theta d x_{2}$

$+d x_{1} \mathrm{~B}^{2} \cos \theta d x_{5}$

$\left.+d x_{2} \mathrm{~B}^{2} \sin \theta d x_{5}\right)\left(X^{1} \frac{\partial}{\partial \mathrm{x}_{1}}+X^{2} \frac{\partial}{\partial \mathrm{x}_{2}}\right.$

$+X^{3} \frac{\partial}{\partial \mathrm{x}_{3}}+X^{4} \frac{\partial}{\partial \mathrm{x}_{4}}+X^{5} \frac{\partial}{\partial \mathrm{x}_{5}}+X^{6} \frac{\partial}{\partial \mathrm{x}_{6}}$

$\left.+X^{7} \frac{\partial}{\partial x_{7}}+X^{8} \frac{\partial}{\partial x_{8}}\right)$

$+\left(d x_{6} \mathrm{~B}^{2} \cos \theta d x_{1}-d x_{6} \mathrm{~B}^{2} \sin \theta d x_{1}\right.$

$-d x_{2} \mathrm{~B}^{2} \cos \theta d x_{6}$

$\left.-d x_{1} \mathrm{~B}^{2} \sin \theta d x_{6}\right)\left(X^{1} \frac{\partial}{\partial \mathrm{x}_{1}}+X^{2} \frac{\partial}{\partial \mathrm{x}_{2}}\right.$

$+X^{3} \frac{\partial}{\partial \mathrm{x}_{3}}+X^{4} \frac{\partial}{\partial \mathrm{x}_{4}}+X^{5} \frac{\partial}{\partial \mathrm{x}_{5}}+X^{6} \frac{\partial}{\partial \mathrm{x}_{6}}$

$\left.+X^{7} \frac{\partial}{\partial \mathrm{x}_{7}}+X^{8} \frac{\partial}{\partial \mathrm{x}_{8}}\right)$

$+\left(-d x_{7} \mathrm{~B}^{2} \cos \theta d x_{3}\right.$

$-d x_{7} \mathrm{~B}^{2} \sin \theta d x_{4}$

$+d x_{3} \mathrm{~B}^{2} \cos \theta d x_{7}$

$\left.+d x_{4} \mathrm{~B}^{2} \sin \theta d x_{7}\right)\left(X^{1} \frac{\partial}{\partial \mathrm{x}_{1}}+X^{2} \frac{\partial}{\partial \mathrm{x}_{2}}\right.$

$+X^{3} \frac{\partial}{\partial \mathrm{x}_{3}}+X^{4} \frac{\partial}{\partial \mathrm{x}_{4}}+X^{5} \frac{\partial}{\partial \mathrm{x}_{5}}+X^{6} \frac{\partial}{\partial \mathrm{x}_{6}}$

$\left.+X^{7} \frac{\partial}{\partial x_{7}}+X^{8} \frac{\partial}{\partial x_{8}}\right)$

$+\left(d x_{8} \mathrm{~B}^{2} \cos \theta d x_{4}-d x_{8} \mathrm{~B}^{2} \sin \theta d x_{3}\right.$

$-d x_{4} \mathrm{~B}^{2} \cos \theta d x_{8}$

$\left.+d x_{3} \mathrm{~B}^{2} \sin \theta d x_{8}\right)\left(X^{1} \frac{\partial}{\partial \mathrm{x}_{1}}+X^{2} \frac{\partial}{\partial \mathrm{x}_{2}}\right.$

$+X^{3} \frac{\partial}{\partial \mathrm{x}_{3}}+X^{4} \frac{\partial}{\partial \mathrm{x}_{4}}+X^{5} \frac{\partial}{\partial \mathrm{x}_{5}}+X^{6} \frac{\partial}{\partial \mathrm{x}_{6}}$

$\left.+X^{7} \frac{\partial}{\partial \mathrm{x}_{7}}+X^{8} \frac{\partial}{\partial \mathrm{x}_{8}}\right)$ 
and so we get

$\frac{1}{2} \quad\left(X^{1} \mathrm{~B}^{2} \cos \theta d x_{5}+X^{1} \mathrm{~B}^{2} \sin \theta d x_{6}+X^{1} \mathrm{~B}^{2} \cos \theta d x_{5}+\right.$ $X^{1} \mathrm{~B}^{2} \sin \theta d x_{6}-X^{2} \mathrm{~B}^{2} \cos \theta d x_{6}-X^{2} \mathrm{~B}^{2} \sin \theta d x_{5}-$ $X^{2} \mathrm{~B}^{2} \cos \theta d x_{6}-X^{2} \mathrm{~B}^{2} \sin \theta d x_{5} \quad+X^{3} \mathrm{~B}^{2} \cos \theta d x_{7}+$ $X^{3} \mathrm{~B}^{2} \sin \theta d x_{8}+X^{3} \mathrm{~B}^{2} \cos \theta d x_{8}+X^{3} \mathrm{~B}^{2} \sin \theta d x_{8}$ $X^{4} \mathrm{~B}^{2} \cos \theta d x_{8}-X^{4} \mathrm{~B}^{2} \sin \theta d x_{7}-X^{4} \mathrm{~B}^{2} \cos \theta d x_{8}-$ $X^{4} \mathrm{~B}^{2} \sin \theta d x_{7}$

$-X^{5} \mathrm{~B}^{-2} \cos \theta d x_{1}+X^{5} \mathrm{~B}^{-2} \sin \theta d x_{2}$ $X^{5} \mathrm{~B}^{-2} \cos \theta d x_{1}+X^{5} \mathrm{~B}^{-2} \sin \theta d x_{2}+X^{6} \mathrm{~B}^{-2} \cos \theta d x_{1}-$ $X^{6} \mathrm{~B}^{-2} \sin \theta d x_{2}+X^{6} \mathrm{~B}^{-2} \cos \theta d x_{1}-X^{6} \mathrm{~B}^{-2} \sin \theta d x_{2}$ $-X^{7} \mathrm{~B}^{-2} \cos \theta d x_{3}-X^{7} \mathrm{~B}^{-2} \sin \theta d x_{4}-X^{7} \mathrm{~B}^{-2} \cos \theta d x_{3}-$ $X^{7} \mathrm{~B}^{-2} \sin \theta d x_{4}+X^{8} \mathrm{~B}^{-2} \cos \theta d x_{4}-X^{8} \mathrm{~B}^{-2} \sin \theta d x_{3}-$ $\left.X^{8} \mathrm{~B}^{-2} \cos \theta d x_{3}+X^{8} \mathrm{~B}^{-2} \sin \theta d x_{4}\right)$

or

$X^{1} \mathrm{~B}^{2} \cos \theta d x_{5}+X^{1} \mathrm{~B}^{2} \sin \theta d x_{6}-X^{2} \mathrm{~B}^{2} \cos \theta d x_{6}$ $X^{2} \mathrm{~B}^{2} \sin \theta d x_{5}-\quad X^{3} \mathrm{~B}^{2} \mathrm{~V} \cos \theta d x_{7}+X^{3} \mathrm{~B}^{2} \sin \theta d x_{8}-$ $X^{4} \mathrm{~B}^{2} \cos \theta d x_{8}-X^{4} \mathrm{~B}^{2} \sin \theta d x_{7}-X^{5} \mathrm{~B}^{-2} \cos \theta d x_{1}+$ $X^{5} \mathrm{~B}^{-2} \sin \theta d x_{2}+X^{6} \mathrm{~B}^{-2} \cos \theta d x_{1}-X^{6} \mathrm{~B}^{-2} \sin \theta d x_{2}+$ $X^{7} \mathrm{~B}^{-2} \cos \theta d x_{3}-X^{7} \mathrm{~B}^{-2} \sin \theta d x_{4}+X^{8} \mathrm{~B}^{-2} \cos \theta d x_{4}-$ $X^{8} \mathrm{~B}^{-2} \sin \theta d x_{3}$

Moreover, the differential of Hamiltonian energy is written as follows:

$\mathrm{dH}$

$=\frac{\partial \mathrm{H}}{\partial \mathrm{x}_{1}} \mathrm{dx}_{1}+\frac{\partial \mathrm{H}}{\partial \mathrm{x}_{2}} \mathrm{dx}_{2}+\frac{\partial \mathrm{H}}{\partial \mathrm{x}_{3}} \mathrm{dx}_{3}+\frac{\partial \mathrm{H}}{\partial \mathrm{x}_{4}} \mathrm{dx}_{4}+\frac{\partial \mathrm{H}}{\partial \mathrm{x}_{5}} \mathrm{dx}_{5}$

$+\frac{\partial \mathrm{H}}{\partial \mathrm{x}_{6}} \mathrm{dx}_{6}+\frac{\partial \mathrm{H}}{\partial \mathrm{x}_{7}} \mathrm{dx} \mathrm{x}_{7}$

$+\frac{\partial \mathrm{H}}{\partial \mathrm{x}_{8}} \mathrm{dx}_{8}$

By means of Eq. (1), using Eqs. (7) and (8), the Hamiltonian vector field is calculated to be

$X^{1} \cos \theta d x_{5}+X^{1} \sin \theta d x_{6}-X^{2} \cos \theta d x_{6}-$ $X^{2} \sin \theta d x_{5}-X^{3} \cos \theta d x_{7}+X^{3} \sin \theta d x_{8}-X^{4} \cos \theta d x_{8}-$ $X^{4} \sin \theta d x_{7}-X^{5} \cos \theta d x_{1}+X^{5} \sin \theta d x_{2}+X^{6} \cos \theta d x_{1}-$ $X^{6} \sin \theta d x_{2}+X^{7} \cos \theta d x_{3}-X^{7} \sin \theta d x_{4}+X^{8} \cos \theta d x_{4}-$ $X^{8} \sin \theta d x_{3}=\frac{\partial \mathrm{H}}{\partial \mathrm{x}_{1}} \mathrm{dx}_{1}+\frac{\partial \mathrm{H}}{\partial \mathrm{x}_{2}} \mathrm{dx}_{2}+\frac{\partial \mathrm{H}}{\partial \mathrm{x}_{3}} \mathrm{dx}_{3}+\frac{\partial \mathrm{H}}{\partial \mathrm{x}_{4}} \mathrm{dx}_{4}+$ $\frac{\partial \mathrm{H}}{\partial \mathrm{x}_{5}} \mathrm{dx}_{5}+\frac{\partial \mathrm{H}}{\partial \mathrm{x}_{6}} \mathrm{dx}_{6}+\frac{\partial \mathrm{H}}{\partial \mathrm{x}_{7}} \mathrm{dx}_{7}+\frac{\partial \mathrm{H}}{\partial \mathrm{x}_{8}} \mathrm{dx}_{8}$

or

$X^{1} \cos \theta d x_{5}+X^{1} \sin \theta d x_{6}-X^{2} \cos \theta d x_{6}-X^{2} \sin \theta d x_{5}$

$+X^{3} \cos \theta d x_{7}+X^{3} \sin \theta d x_{8}$

$-X^{4} \cos \theta d x_{8}$

$-X^{4} \sin \theta d x_{7}-X^{5} \cos \theta d x_{2}$

$+X^{5} \sin \theta d x_{1} X^{6} \cos \theta d x_{1}$

$-X^{6} \sin \theta d x_{2}+X^{6} \cos \theta d x_{2}$

$-X^{7} \cos \theta d x_{3}$

$-X^{7} \sin \theta d x_{4}-X^{8} \cos \theta d x_{4}$

$-X^{8} \sin \theta d x_{3}$

$=\frac{\partial \mathrm{H}}{\partial \mathrm{x}_{1}} \mathrm{dx}_{1}+\frac{\partial \mathrm{H}}{\partial \mathrm{x}_{2}} \mathrm{dx}_{2}+\frac{\partial \mathrm{H}}{\partial \mathrm{x}_{3}} \mathrm{dx}_{3}$

$+\frac{\partial \mathrm{H}}{\partial \mathrm{x}_{4}} \mathrm{dx}_{4}+\frac{\partial \mathrm{H}}{\partial \mathrm{x}_{5}} \mathrm{dx}_{5}+\frac{\partial \mathrm{H}}{\partial \mathrm{x}_{6}} \mathrm{dx}_{6}$

$+\frac{\partial \mathrm{H}}{\partial \mathrm{x}_{7}} \mathrm{dx}_{7}+\frac{\partial \mathrm{H}}{\partial \mathrm{x}_{8}} \mathrm{dx}_{8}$
By comparing the two sides we get the following: $X^{5} \mathrm{~B}^{-2} \cos \theta d x_{1}+X^{6} \mathrm{~B}^{-2} \sin \theta d x_{1}=\mathrm{B}^{-2}\left(X^{5} \cos \theta\right.$

$$
\begin{aligned}
& \left.+X^{6} \sin \theta\right) \mathrm{dx}_{1}=\frac{\partial \mathrm{H}}{\partial \mathrm{x}_{1}} \mathrm{dx}_{1} \\
& \Rightarrow \mathrm{B}^{-2}\left(X^{5} \cos \theta+X^{6} \sin \theta\right)=\frac{\partial \mathrm{H}}{\partial \mathrm{x}_{1}}
\end{aligned}
$$

$X^{5} \mathrm{~B}^{-2} \sin \theta d x_{2}-X^{6} \mathrm{~B}^{-2} \cos \theta d x_{2}$

$=\mathrm{B}^{-2}\left(X^{5} \sin \theta-X^{6} \cos \theta\right) d x_{2}$

$=\frac{\partial \mathrm{H}}{\partial \mathrm{x}_{2}} \mathrm{dx}_{2} \Rightarrow \mathrm{B}^{-2}\left(X^{5} \sin \theta-X^{6} \cos \theta\right)$

$=\frac{\partial \mathrm{H}}{\partial \mathrm{x}_{2}}$

$-X^{7} \mathrm{~B}^{-2} \cos \theta d x_{3}+X^{8} \mathrm{~B}^{-2} \sin \theta d x_{3}$

$=\mathrm{B}^{-2}\left(-X^{7} \cos \theta+X^{8} \sin \theta\right) d x_{3}$

$=\frac{\partial \mathrm{H}}{\partial \mathrm{x}_{3}} \mathrm{dx}_{3} \Rightarrow \mathrm{B}^{-2}\left(-X^{7} \cos \theta+X^{8} \sin \theta\right)$

$=\frac{\partial \mathrm{H}}{\partial \mathrm{x}_{3}}$

$X^{7} \mathrm{~B}^{-2} \sin \theta d x_{4}-X^{8} \mathrm{~B}^{-2} \cos \theta d x_{4}$

$=\mathrm{B}^{-2}\left(X^{7} \sin \theta-X^{8} \cos \theta\right) d x_{4}$

$=\frac{\partial \mathrm{H}}{\partial \mathrm{x}_{4}} \mathrm{dx}_{4} \Rightarrow \mathrm{B}^{-2}\left(X^{7} \sin \theta-X^{8} \cos \theta\right)$

$=\frac{\partial \mathrm{H}}{\partial \mathrm{x}_{4}}$

$-X^{1} \mathrm{~B}^{2} \cos \theta d x_{5}-X^{2} \mathrm{~B}^{2} \sin \theta d x_{5}$

$=\mathrm{B}^{2}\left(-X^{1} \cos \theta-X^{2} \sin \theta\right) d x_{5}$

$=\frac{\partial \mathrm{H}}{\partial \mathrm{x}_{5}} \mathrm{dx}_{5}$

$\Rightarrow \mathrm{B}^{2}\left(-X^{1} \cos \theta-X^{2} \sin \theta\right)=\frac{\partial \mathrm{H}}{\partial \mathrm{x}_{5}}$

$-X^{1} \mathrm{~B}^{2} \sin \theta d x_{6}+X^{2} \mathrm{~B}^{2} \mathrm{~V} \cos \theta d x_{6}$

$=\mathrm{B}^{2}\left(-X^{1} \sin \theta+X^{2} \cos \theta\right) d x_{6}$

$=\frac{\partial \mathrm{H}}{\partial \mathrm{x}_{6}} \mathrm{dx}_{6} \Rightarrow \mathrm{B}^{2}\left(-X^{1} \sin \theta+X^{2} \cos \theta\right)$

$=\frac{\partial \mathrm{H}}{\partial \mathrm{x}_{6}}$

$-X^{3} \mathrm{~B}^{2} \cos \theta d x_{7}-X^{4} \mathrm{~B}^{2} \sin \theta d x_{7}$

$=\mathrm{B}^{2}\left(-X^{3} \cos \theta-X^{4} \sin \theta\right) d x_{7}$

$=\frac{\partial \mathrm{H}}{\partial \mathrm{x}_{7}} \mathrm{dx}_{7} \Rightarrow \mathrm{B}^{2}\left(-X^{3} \sin \theta+X^{4} \cos \theta\right)$

$=\frac{\partial \mathrm{H}}{\partial \mathrm{x}_{7}}$

$-X^{3} \mathrm{~B}^{2} \sin \theta d x_{8}+X^{4} \mathrm{~B}^{2} \cos \theta d x_{8}$

$=\mathrm{B}^{2}\left(-X^{3} \sin \theta+X^{4} \cos \theta\right) d x_{8}$

$=\frac{\partial \mathrm{H}}{\partial \mathrm{x}_{7}} \mathrm{dx}_{7} \Rightarrow \mathrm{B}^{2}\left(-X^{3} \sin \theta+X^{4} \cos \theta\right)$

$=\frac{\partial \mathrm{H}}{\partial \mathrm{x}_{8}}$

So we find that

$$
\begin{aligned}
X^{1} & =-\mathrm{B}^{2} \cos \theta \frac{\partial \mathrm{H}}{\partial \mathrm{x}_{5}}-\mathrm{B}^{2} \sin \theta \frac{\partial \mathrm{H}}{\partial \mathrm{x}_{6}} \\
X^{2} & =\mathrm{B}^{2} \cos \theta \frac{\partial \mathrm{H}}{\partial \mathrm{x}_{6}}-\mathrm{B}^{2} \sin \theta \frac{\partial \mathrm{H}}{\partial \mathrm{x}_{5}} \\
X^{3} & =-\mathrm{B}^{2} \cos \theta \frac{\partial \mathrm{H}}{\partial \mathrm{x}_{7}}-\mathrm{B}^{2} \sin \theta \frac{\partial \mathrm{H}}{\partial \mathrm{x}_{8}} \\
X^{4} & =\mathrm{B}^{2} \cos \theta \frac{\partial \mathrm{H}}{\partial \mathrm{x}_{8}}-\mathrm{B}^{2} \sin \theta \frac{\partial \mathrm{H}}{\partial \mathrm{x}_{7}} \\
X^{5} & =\mathrm{B}^{-2} \cos \theta \frac{\partial \mathrm{H}}{\partial \mathrm{x}_{1}}+\mathrm{B}^{-2} \sin \theta \frac{\partial \mathrm{H}}{\partial \mathrm{x}_{2}}
\end{aligned}
$$




$$
\begin{array}{r}
X^{6}=-\mathrm{B}^{-2} \cos \theta \frac{\partial \mathrm{H}}{\partial \mathrm{x}_{2}}+\mathrm{B}^{-2} \sin \theta \frac{\partial \mathrm{H}}{\partial \mathrm{x}_{1}} \\
X^{7}=\mathrm{B}^{-2} \cos \theta \frac{\partial \mathrm{H}}{\partial \mathrm{x}_{3}}+\mathrm{B}^{-2} \sin \theta \frac{\partial \mathrm{H}}{\partial \mathrm{x}_{4}} \\
X^{8}=-\mathrm{B}^{-2} \cos \theta \frac{\partial \mathrm{H}}{\partial \mathrm{x}_{4}}+\mathrm{B}^{-2} \sin \theta \frac{\partial \mathrm{H}}{\partial \mathrm{x}_{3}}
\end{array}
$$

Equation compensate (11) in equation (6) we get

$$
\begin{aligned}
& \mathrm{B}^{2}\left(-\cos \theta \frac{\partial \mathrm{H}}{\partial \mathrm{x}_{5}}-\sin \theta \frac{\partial \mathrm{H}}{\partial \mathrm{x}_{6}}\right) \frac{\partial}{\partial \mathrm{x}_{1}}+\mathrm{B}^{2}\left(\cos \theta \frac{\partial \mathrm{H}}{\partial \mathrm{x}_{6}}-\right. \\
& \left.\sin \theta \frac{\partial \mathrm{H}}{\partial \mathrm{x}_{5}}\right) \frac{\partial}{\partial \mathrm{x}_{2}}+\mathrm{B}^{2}\left(-\cos \theta \frac{\partial \mathrm{H}}{\partial \mathrm{x}_{7}}-\sin \theta \frac{\partial \mathrm{H}}{\partial \mathrm{x}_{8}}\right) \frac{\partial}{\partial \mathrm{x}_{3}}+ \\
& \mathrm{B}^{2}\left(\cos \theta \frac{\partial \mathrm{H}}{\partial \mathrm{x}_{8}}-\sin \theta \frac{\partial \mathrm{H}}{\partial \mathrm{x}_{7}}\right) \frac{\partial}{\partial \mathrm{x}_{4}}+\mathrm{B}^{2}\left(\cos \theta \frac{\partial \mathrm{H}}{\partial \mathrm{x}_{1}}+\right. \\
& \left.\sin \theta \frac{\partial \mathrm{H}}{\partial \mathrm{x}_{2}}\right) \frac{\partial}{\partial \mathrm{x}_{5}}+\mathrm{B}^{-2}\left(-\cos \theta \frac{\partial \mathrm{H}}{\partial \mathrm{x}_{2}}+\sin \theta \frac{\partial \mathrm{H}}{\partial \mathrm{x}_{1}}\right) \frac{\partial}{\partial \mathrm{x}_{6}}+ \\
& \mathrm{B}^{-2}\left(\cos \theta \frac{\partial \mathrm{H}}{\partial \mathrm{x}_{3}}+\sin \theta \frac{\partial \mathrm{H}}{\partial \mathrm{x}_{4}}\right) \frac{\partial}{\partial \mathrm{x}_{7}}+\mathrm{B}^{-2}\left(-\cos \theta \frac{\partial \mathrm{H}}{\partial \mathrm{x}_{4}}+\right. \\
& \left.\sin \theta \frac{\partial \mathrm{H}}{\partial \mathrm{x}_{3}}\right) \frac{\partial}{\partial \mathrm{x}_{8}}=\frac{\partial \mathrm{H}}{\partial \mathrm{x}_{1}} \mathrm{dx}_{1}+\frac{\partial \mathrm{H}}{\partial \mathrm{x}_{2}} \mathrm{dx}_{2}+\frac{\partial \mathrm{H}}{\partial \mathrm{x}_{3}} \mathrm{dx}_{3}+\frac{\partial \mathrm{H}}{\partial \mathrm{x}_{4}} \mathrm{dx}_{4}+ \\
& \frac{\partial \mathrm{H}}{\partial \mathrm{x}_{5}} \mathrm{dx}_{5}+\frac{\partial \mathrm{H}}{\partial \mathrm{x}_{6}} \mathrm{dx}_{6}+\frac{\partial \mathrm{H}}{\partial \mathrm{x}_{7}} \mathrm{dx}_{7}+ \\
& \frac{\partial \mathrm{H}}{\partial \mathrm{x}_{8}} \mathrm{dx}_{8}
\end{aligned}
$$

Suppose that a curve $\alpha: \mathrm{I} \subset \mathrm{R} \rightarrow \mathrm{T}^{*} \mathcal{M}=R^{2 n}$ is an integral curve of the Hamiltonian vector field $\mathrm{X}_{\mathrm{H}}$, i.e.,

$X_{H}(\alpha(t))=\frac{d \alpha(t)}{d t} \quad, \quad t \in I$.

In the local coordinates, if it is considered to be

$\alpha(\mathrm{t})$

$=\left(\mathrm{x}_{1}(\mathrm{t}), \mathrm{x}_{2}(\mathrm{t}), \mathrm{x}_{2}(\mathrm{t}), \mathrm{x}_{4}(\mathrm{t}), \mathrm{x}_{5}(\mathrm{t}), \mathrm{x}_{6}(\mathrm{t}), \mathrm{x}_{7}(\mathrm{t}), \mathrm{x}_{8}(\mathrm{t})\right)$

We obtain

$$
\begin{aligned}
\frac{\mathrm{d} \alpha(\mathrm{t})}{\mathrm{dt}}=\frac{\mathrm{dx}_{1}}{\mathrm{dt}} \frac{\partial}{\partial \mathrm{x}_{1}}+ & \frac{\mathrm{dx}_{2}}{\mathrm{dt}} \frac{\partial}{\partial \mathrm{x}_{2}}+\frac{\mathrm{dx}_{3}}{\mathrm{dt}} \frac{\partial}{\partial \mathrm{x}_{3}}+\frac{\mathrm{dx}_{4}}{\mathrm{dt}} \frac{\partial}{\partial \mathrm{x}_{4}}+\frac{\mathrm{dx_{5 }}}{\mathrm{dt}} \frac{\partial}{\partial \mathrm{x}_{5}} \\
& +\frac{\mathrm{dx}_{6}}{\mathrm{dt}} \frac{\partial}{\partial \mathrm{x}_{6}}+\frac{\mathrm{dx}_{7}}{\mathrm{dt}} \frac{\partial}{\partial \mathrm{x}_{7}} \\
& +\frac{\mathrm{dx}_{8}}{\mathrm{dt}} \frac{\partial}{\partial \mathrm{x}_{8}}
\end{aligned}
$$

Taking the equation $(12)=$ the equation (13)

$$
\begin{aligned}
& \mathrm{B}^{2}\left(-\cos \theta \frac{\partial \mathrm{H}}{\partial \mathrm{x}_{5}}-\sin \theta \frac{\partial \mathrm{H}}{\partial \mathrm{x}_{6}}\right) \frac{\partial}{\partial \mathrm{x}_{1}} \\
& +\mathrm{B}^{2}\left(\cos \theta \frac{\partial \mathrm{H}}{\partial \mathrm{x}_{6}}-\sin \theta \frac{\partial \mathrm{H}}{\partial \mathrm{x}_{5}}\right) \frac{\partial}{\partial \mathrm{x}_{2}} \\
& +\mathrm{B}^{2}\left(-\cos \theta \frac{\partial \mathrm{H}}{\partial \mathrm{x}_{7}}-\sin \theta \frac{\partial \mathrm{H}}{\partial \mathrm{x}_{8}}\right) \frac{\partial}{\partial \mathrm{x}_{3}} \\
& +\mathrm{B}^{2}\left(\cos \theta \frac{\partial \mathrm{H}}{\partial \mathrm{x}_{8}}-\sin \theta \frac{\partial \mathrm{H}}{\partial \mathrm{x}_{7}}\right) \frac{\partial}{\partial \mathrm{x}_{4}} \\
& +\mathrm{B}^{-2}\left(\cos \theta \frac{\partial \mathrm{H}}{\partial \mathrm{x}_{1}}+\sin \theta \frac{\partial \mathrm{H}}{\partial \mathrm{x}_{2}}\right) \frac{\partial}{\partial \mathrm{x}_{5}} \\
& +\mathrm{B}^{-2}\left(-\cos \theta \frac{\partial \mathrm{H}}{\partial \mathrm{x}_{2}}+\sin \theta \frac{\partial \mathrm{H}}{\partial \mathrm{x}_{1}}\right) \frac{\partial}{\partial \mathrm{x}_{6}} \\
& +\mathrm{B}^{-2}\left(\cos \theta \frac{\partial \mathrm{H}}{\partial \mathrm{x}_{3}}+\sin \theta \frac{\partial \mathrm{H}}{\partial \mathrm{x}_{4}}\right) \frac{\partial}{\partial \mathrm{x}_{7}} \\
& +\mathrm{B}^{-2}\left(-\cos \theta \frac{\partial \mathrm{H}}{\partial \mathrm{x}_{4}}+\sin \theta \frac{\partial \mathrm{H}}{\partial \mathrm{x}_{3}}\right) \frac{\partial}{\partial \mathrm{x}_{8}} \\
& =\frac{\mathrm{dx}_{1}}{\mathrm{dt}} \frac{\partial}{\partial \mathrm{x}_{1}}+\frac{\mathrm{dx}_{2}}{\mathrm{dt}} \frac{\partial}{\partial \mathrm{x}_{2}}+\frac{\mathrm{dx}_{3}}{\mathrm{dt}} \frac{\partial}{\partial \mathrm{x}_{3}}+\frac{\mathrm{dx}_{4}}{\mathrm{dt}} \frac{\partial}{\partial \mathrm{x}_{4}} \\
& +\frac{\mathrm{dx}_{5}}{\mathrm{dt}} \frac{\partial}{\partial \mathrm{x}_{5}}+\frac{\mathrm{dx}_{6}}{\mathrm{dt}} \frac{\partial}{\partial \mathrm{x}_{6}}+\frac{\mathrm{dx}_{7}}{\mathrm{dt}} \frac{\partial}{\partial \mathrm{x}_{7}}+\frac{\mathrm{dx}_{8}}{\mathrm{dt}} \frac{\partial}{\partial \mathrm{x}_{8}}
\end{aligned}
$$

By comparing the two sides of the equation we get the

$$
\begin{gathered}
\mathrm{B}^{2}\left(-\cos \theta \frac{\partial \mathrm{H}}{\partial \mathrm{x}_{5}}-\sin \theta \frac{\partial \mathrm{H}}{\partial \mathrm{x}_{6}}\right) \frac{\partial}{\partial \mathrm{x}_{1}}=\frac{\mathrm{dx}_{1}}{\mathrm{dt}} \frac{\partial}{\partial \mathrm{x}_{1}} \\
-\mathrm{B}^{2} \cos \theta \frac{\partial \mathrm{H}}{\partial \mathrm{x}_{5}}-\mathrm{B}^{2} \sin \theta \frac{\partial \mathrm{H}}{\partial \mathrm{x}_{6}}=\frac{\mathrm{dx}_{1}}{\mathrm{dt}}
\end{gathered}
$$

and

$$
\begin{gathered}
\mathrm{B}^{2}\left(\cos \theta \frac{\partial \mathrm{H}}{\partial \mathrm{x}_{6}}-\sin \theta \frac{\partial \mathrm{H}}{\partial \mathrm{x}_{5}}\right) \frac{\partial}{\partial \mathrm{x}_{2}}=\frac{\mathrm{dx}_{2}}{\mathrm{dt}} \frac{\partial}{\partial \mathrm{x}_{2}} \\
\mathrm{~B}^{2} \cos \theta \frac{\partial \mathrm{H}}{\partial \mathrm{x}_{6}}-\mathrm{B}^{2} \sin \theta \frac{\partial \mathrm{H}}{\partial \mathrm{x}_{5}}=\frac{\mathrm{dx}_{2}}{\mathrm{dt}}
\end{gathered}
$$

and

$$
\begin{gathered}
\mathrm{B}^{2}\left(-\cos \theta \frac{\partial \mathrm{H}}{\partial \mathrm{x}_{7}}-\sin \theta \frac{\partial \mathrm{H}}{\partial \mathrm{x}_{8}}\right) \frac{\partial}{\partial \mathrm{x}_{3}}=\frac{\mathrm{dx_{3 }}}{\mathrm{dt}} \frac{\partial}{\partial \mathrm{x}_{3}} \\
-\mathrm{B}^{2} \cos \theta \frac{\partial \mathrm{H}}{\partial \mathrm{x}_{7}}-\mathrm{B}^{2} \sin \theta \frac{\partial \mathrm{H}}{\partial \mathrm{x}_{8}}=\frac{\mathrm{dx}_{3}}{\mathrm{dt}}
\end{gathered}
$$

and

$$
\begin{gathered}
\mathrm{B}^{2}\left(\cos \theta \frac{\partial \mathrm{H}}{\partial \mathrm{x}_{8}}-\sin \theta \frac{\partial \mathrm{H}}{\partial \mathrm{x}_{7}}\right) \frac{\partial}{\partial \mathrm{x}_{4}}=\frac{\mathrm{dx}_{4}}{\mathrm{dt}} \frac{\partial}{\partial \mathrm{x}_{4}} \\
\mathrm{~B}^{2} \cos \theta \frac{\partial \mathrm{H}}{\partial \mathrm{x}_{8}}-\mathrm{B}^{2} \sin \theta \frac{\partial \mathrm{H}}{\partial \mathrm{x}_{7}}=\frac{\mathrm{dx}_{4}}{\mathrm{dt}}
\end{gathered}
$$

and

$$
\begin{gathered}
\mathrm{B}^{-2}\left(\cos \theta \frac{\partial \mathrm{H}}{\partial \mathrm{x}_{1}}+\sin \theta \frac{\partial \mathrm{H}}{\partial \mathrm{x}_{2}}\right) \frac{\partial}{\partial \mathrm{x}_{5}}=\frac{\mathrm{dx}_{5}}{\mathrm{dt}} \frac{\partial}{\partial \mathrm{x}_{5}} \\
\mathrm{~B}^{-2} \cos \theta \frac{\partial \mathrm{H}}{\partial \mathrm{x}_{1}}+\mathrm{B}^{-2} \sin \theta \frac{\partial \mathrm{H}}{\partial \mathrm{x}_{2}}=\frac{\mathrm{dx}_{5}}{\mathrm{dt}}
\end{gathered}
$$

and

$$
\begin{aligned}
\mathrm{B}^{-2}\left(-\cos \theta \frac{\partial \mathrm{H}}{\partial \mathrm{x}_{2}}+\sin \theta \frac{\partial \mathrm{H}}{\partial \mathrm{x}_{1}}\right) \frac{\partial}{\partial \mathrm{x}_{6}} & =\frac{\mathrm{dx}_{6}}{\mathrm{dt}} \frac{\partial}{\partial \mathrm{x}_{6}} \\
-\mathrm{B}^{-2} \cos \theta \frac{\partial \mathrm{H}}{\partial \mathrm{x}_{2}}+\mathrm{B}^{-2} \sin \theta \frac{\partial \mathrm{H}}{\partial \mathrm{x}_{1}} & =\frac{\mathrm{dx}_{6}}{\mathrm{dt}}
\end{aligned}
$$

and

$$
\begin{gathered}
\mathrm{B}^{-2}\left(\cos \theta \frac{\partial \mathrm{H}}{\partial \mathrm{x}_{3}}+\sin \theta \frac{\partial \mathrm{H}}{\partial \mathrm{x}_{4}}\right) \frac{\partial}{\partial \mathrm{x}_{7}}=\frac{\mathrm{dx}_{7}}{\mathrm{dt}} \frac{\partial}{\partial \mathrm{x}_{7}} \\
\mathrm{~B}^{-2} \cos \theta \frac{\partial \mathrm{H}}{\partial \mathrm{x}_{3}}+\mathrm{B}^{-2} \sin \theta \frac{\partial \mathrm{H}}{\partial \mathrm{x}_{4}}=\frac{\mathrm{dx}_{7}}{\mathrm{dt}}
\end{gathered}
$$

and

$$
\begin{gathered}
\mathrm{B}^{-2}\left(-\cos \theta \frac{\partial \mathrm{H}}{\partial \mathrm{x}_{4}}+\mathrm{B}^{2} \sin \theta \frac{\partial \mathrm{H}}{\partial \mathrm{x}_{3}}\right) \frac{\partial}{\partial \mathrm{x}_{8}}=\frac{\mathrm{dx}_{8}}{\mathrm{dt}} \frac{\partial}{\partial \mathrm{x}_{8}} \\
-\mathrm{B}^{-2} \cos \theta \frac{\partial \mathrm{H}}{\partial \mathrm{x}_{4}}+\mathrm{B}^{-2} \sin \theta \frac{\partial \mathrm{H}}{\partial \mathrm{x}_{3}}=\frac{\mathrm{dx}_{8}}{\mathrm{dt}}
\end{gathered}
$$

Thus Hamilton's equations are

$$
\begin{aligned}
-\mathrm{B}^{2} \cos \theta \frac{\partial \mathrm{H}}{\partial \mathrm{x}_{5}}-\mathrm{B}^{2} & \sin \theta \frac{\partial \mathrm{H}}{\partial \mathrm{x}_{6}} \\
& =\frac{\mathrm{dx}_{1}}{\mathrm{dt}}, \quad \mathrm{B}^{2} \cos \theta \frac{\partial \mathrm{H}}{\partial \mathrm{x}_{6}}-\mathrm{B}^{2} \sin \theta \frac{\partial \mathrm{H}}{\partial \mathrm{x}_{5}} \\
& =\frac{\mathrm{dx}_{2}}{\mathrm{dt}}
\end{aligned}
$$




$$
\begin{aligned}
& -\mathrm{B}^{2} \cos \theta \frac{\partial \mathrm{H}}{\partial \mathrm{x}_{7}}-\mathrm{B}^{2} \sin \theta \frac{\partial \mathrm{H}}{\partial \mathrm{x}_{8}} \\
& =\frac{\mathrm{dx}_{3}}{\mathrm{dt}} \quad, \quad \mathrm{B}^{2} \cos \theta \frac{\partial \mathrm{H}}{\partial \mathrm{x}_{8}} \\
& -\mathrm{B}^{2} \sin \theta \frac{\partial \mathrm{H}}{\partial \mathrm{x}_{7}}=\frac{\mathrm{dx}_{4}}{\mathrm{dt}} \\
& \mathrm{B}^{-2} \cos \theta \frac{\partial \mathrm{H}}{\partial \mathrm{x}_{1}}+\mathrm{B}^{2} \sin \theta \frac{\partial \mathrm{H}}{\partial \mathrm{x}_{2}} \\
& =\frac{\mathrm{dx}_{5}}{\mathrm{dt}} \quad,-\mathrm{B}^{2-} \cos \theta \frac{\partial \mathrm{H}}{\partial \mathrm{x}_{2}} \\
& +\mathrm{B}^{-2} \sin \theta \frac{\partial \mathrm{H}}{\partial \mathrm{x}_{1}}=\frac{\mathrm{dx}_{6}}{\mathrm{dt}} \\
& \mathrm{B}^{-2} \cos \theta \frac{\partial \mathrm{H}}{\partial \mathrm{x}_{3}}+\mathrm{B}^{-2} \sin \theta \frac{\partial \mathrm{H}}{\partial \mathrm{x}_{4}} \\
& =\frac{\mathrm{dx}_{7}}{\mathrm{dt}},-\mathrm{B}^{-2} \cos \theta \frac{\partial \mathrm{H}}{\partial \mathrm{x}_{4}}+\mathrm{B}^{-2} \sin \theta \frac{\partial \mathrm{H}}{\partial \mathrm{x}_{3}} \\
& =\frac{\mathrm{dx}_{8}}{\mathrm{dt}}
\end{aligned}
$$

Hence the triple $\left(\mathrm{T}^{*} \mathcal{M}=\mathcal{M}^{9}, \phi_{\mathrm{H}}, \mathrm{X}_{\mathrm{H}}\right)$ is shown to be a Hamiltonian mechanical system which are deduced by means of an almost real structure $j^{*}$ and using of basis $\left\{\frac{\partial}{\partial \mathrm{x}_{\mathrm{i}}}: \mathrm{i}=\right.$ $1,2,3,4,5,6,7,8\}$ on the distributions $\mathrm{T}^{*} \mathcal{M}$

\section{Conclusions}

The solutions of the Hamiltonian equations determined by (14) on the mechanical system on 9-contact manifolds $\left(\mathcal{M}^{9}, \omega, \mathrm{X}_{\mathrm{H}}\right)$ are the paths of vector field $\mathrm{X}_{\mathrm{H}}$ on $\mathcal{M}^{9}$. To construct Hamilton's canonical equations for a mechanical system proceed as follows: 1. Choose your generalized coordinates $\mathrm{q}=(\mathrm{q} 1, \ldots, \mathrm{qn}) \mathrm{T}$ and construct.

2. Define and compute the generalized momenta

3. Construct and compute the Hamiltonian function

4. Write down Hamilton's equations of motion

\section{Ethical issue}

Authors are aware of, and comply with, best practice in publication ethics specifically with regard to authorship (avoidance of guest authorship), dual submission, manipulation of figures, competing interests and compliance with policies on research ethics. Authors adhere to publication requirements that submitted work is original and has not been published elsewhere in any language.

\section{Competing interests}

The authors declare that there is no conflict of interest that would prejudice the impartiality of this scientific work.

\section{Authors' contribution}

All authors of this study have a complete contribution for data collection, data analyses and manuscript writing.

\section{References}

[1] Thall E. Thall's History of Quantum Mechanics. 2011.

[2] Tekkoyun M. Lagrangian and Hamiltonian Dynamics on ParaKählerian Space Form. arXiv preprint arXiv:0902.4522. 2009 Feb 26.

[3] KASAP Z. Hamilton Equations on a Contact 5-Manifolds. Elixir International Journal, Math. 2016 Mar 1;92:38743-8.

[4] Kasap Z. Conformal E Lagrange Mechanical Equations on Contact 5-Manifolds. international Journal of Innovative Mathematical Research.;3.

[5] Tekkoyun M, Yayli Y. Mechanical systems on generalizedquaternionic Kähler manifolds. International Journal of Geometric Methods in Modern Physics. 2011 Nov;8(07):1419-31.

[6] Cannas da Silva A. Introduction to symplectic and Hamiltonian geometry.

[7] Burkard E. From Classical Mechanics to Symplectic Geometry. 2013.

[8] Hofer H, Zehnder E. Symplectic invariants and Hamiltonian dynamics. InThe Floer memorial volume 1995 (pp. 525-544). Birkhäuser Basel.

[9] Tekkoyun M, Yayli Y. Mechanical systems on generalizedquaternionic Kähler manifolds. International Journal of Geometric Methods in Modern Physics. 2011 Nov;8(07):1419-31.

[10] Ata E, Yaylı Y. A global condition for the triviality of an almost split quaternionic structure on split complex manifolds. Int. J. Math. Sci.(WASET). 2008 Jul 28;2(1):47-51

[11] Perko L. Hamiltonian Systems with Two-Degrees of Freedom. Differential equations and dynamical systems. 3rd ed. New York: Springer. 2001:228-237.

[13] Zaslavsky GM, Zaslavskij GM. Hamiltonian chaos and fractional dynamics. Oxford University Press on Demand; 2005.

[14] Bellettini C. Almost complex structures and calibrated integra cycles in contact 5-manifolds. Advances in Calculus of Variations. 2013 Jul 1;6(3):339-74.

[15] Kodama H. Complex contact three manifolds with Legendrian vector fields. Proceedings of the Japan Academy, Series A Mathematical Sciences. 2002;78(4):51-4

[16] Newlander A, Nirenberg L. Complex analytic coordinates in almost complex manifolds. Annals of Mathematics. 1957 May $1: 391-404$

[17] Brozos-Vázquez M, Fiedler B, García-Río E, Gilkey P, Nikcevic S, Stanilov G, Tsankov Y, Vázquez-Lorenzo R, Videv V. StanilovTsankov-Videv Theory. SIGMA. Symmetry, Integrability and Geometry: Methods and Applications. 2007 Sep 28;3:095.

[18] Davidov J, Díaz-Ramos JC, García-Río E, Matsushita Y, Muškarov O, Vázquez-Lorenzo R. Almost Kähler Walker 4manifolds. Journal of Geometry and Physics. 2007 Feb 1;57(3):1075-88

[19] Tekkoyun M. A Survey on Geometric Dynamics of 4-Walker Manifold. Journal of Modern Physics. 2011 Nov 10;2(11):1318 23.

[20] Chaubey SK. Almost contact metric manifolds admitting semisymmetric non-metric connection. Bulletin of Mathematical Analysis and Applications. 2011 Sep 1;3(2):252-60.

[21] Dwivedi MK, Jun JB, Tripathi MM. On endo curvature tensor of a contact metric manifold. Tamkang Journal of Mathematics. 2008 Jun 30;39(2):177-86

[22] Malek F, Balgeshir MB. Slant submanifolds of almost contact metric 3-structure manifolds. Mediterranean journal of mathematics. 2013 May 1;10(2):1023-33. 